\title{
Preparation of silver powder through glycerol process
}

\author{
AMIT SINHA* and B P SHARMA \\ Powder Metallurgy Division, Bhabha Atomic Research Centre, Vashi Complex, Navi Mumbai 400 705, India
}

MS received 6 November 2004; revised 8 April 2005

\begin{abstract}
High purity fine silver powder with uniform particle morphology was prepared through glycerol process. The process involves reduction of silver nitrate by glycerol under atmospheric conditions at a temperature below $175^{\circ} \mathrm{C}$. Glycerol, in this process, acts as a solvent as well as a reducing agent. The powders prepared through this process were characterized by X-ray diffraction (XRD), scanning electron microscopy (SEM) and chemical analysis. The powders were well crystalline and contained oxygen, carbon and hydrogen as impurities. Overall purity was better than $99.9 \%$. The yield of silver powder was better than $99 \%$.
\end{abstract}

Keywords. Silver powder; glycerol process.

\section{Introduction}

Silver powder finds extensive applications in electronic industry, particularly in the field for making conducting inks and paste for thin/thick films. Conductive silver paste forms the basis for producing electronic components such as hybrid microcircuits and the internal electrodes of multi-layer ceramic capacitors (Larry et al 1980). For such applications, silver powder of specific morphology is desired. The powders should be composed of crystalline non-agglomerated micron/submicron particles with narrow size distribution.

Till date, silver powder has been prepared through various processes ranging from chemical, physical (atomization and milling), and electrochemical to thermal decomposition. Every process produces powders with characteristic morphology and purity that ultimately govern its functional properties. Among the various preparative methods used, chemical processes offer distinct advantage over the others in terms of powder morphology as well as efficient scale-up for mass production. There have been a number of reports that describe the synthesis of silver powder through chemical processes. These include reduction of silver salts by $\mathrm{NaBH}_{4}, \mathrm{HCHO} / \mathrm{NaOH} /$ $\mathrm{Na}_{2} \mathrm{CO}_{3}$ (Duff et al 1993; Burst et al 1994; Rao 1994; Isabel and Luis 1999; Bonet et al 2000; Chou and Ren 2000). The reduction of silver chloride by glucose has also been reported (Brauser 1965).

The polyol process has been successfully employed for preparation of metal powders belonging to groups VII and IB (Fievet et al 1989). In this process, a suitable solid inorganic/organic salt of metal is suspended in a liquid polyol, the suspension is stirred and heated to a given temperature. The reduction of metallic salt by polyol

*Author for correspondence (amit97@yahoo.com) quantitatively yields metal powder in a finely dispersed form. The control of particle morphology is facilitated by the kinetic control of the nucleation and growth steps. Definite separation between the nucleation and growth stages is a prerequisite for the formation of mono-sized particle (Fievet et al 1989). One of the measures to separate growth from nucleation may be the addition of seed particles where seed crystals act as nuclei and homogeneous nucleation is thus replaced by heterogeneous nucleation.

The preparation of metal powders through polyol process has largely been focused on the use of ethylene glycol or di-ethelene glycol or their mixtures as reducing agent and solvent. The limitation of ethylene glycol as reducing agent, in some cases is being compensated by the use of additional reducing agent in the form of polyvinylpyrrolidone (PVP) (Silvert et al 1997). Recently, Sinha and Sharma (2002) described a process for preparation of copper powder using glycerol. Glycerol has higher boiling point $\left(290^{\circ} \mathrm{C}\right)$ as compared with that of ethylene glycol $\left(197 \cdot 3^{\circ} \mathrm{C}\right)$ and hence it facilitates a higher reaction temperature. Though silver powder has been prepared through polyol route using ethylene glycol and PVP as reducing agents, the use of glycerol to prepare silver powder has not been studied. In the present investigation, silver powder has been prepared starting from silver nitrate using glycerol as solvent and reducing agent.

\section{Experimental}

Silver nitrate (AR grade, E-Merck India Ltd) was used as starting material for the preparation of silver powder. A predetermined amount of starting salt was suspended in glycerol (AR grade). The molar ratio $(R)$ between silver nitrate and glycerol was varied from $0 \cdot 01-0 \cdot 1$. No protective agent in the form of PVP or seed particle (nucleating 
agent) was used. The suspension was heated, while stirring, on a laboratory hot plate. The reaction temperature was measured using a thermometer submerged into the solution through a glass port. The silver nitrate salt started to dissolve with increase in temperature. After about one hour of incubation time, silver powder started to precipitate.

After the precipitation, the suspension of silver powder obtained was naturally cooled to room temperature and then diluted with ethanol. The metal particles were recovered from the mother solution by repeated centrifugation followed by washing with ethanol. The powder obtained was dried under vacuum at $80^{\circ} \mathrm{C}$ for $1 \mathrm{~h}$.

The phase analysis of the dried powder was carried out by X-ray diffraction (Philips Diffractometer, Model PW1710) using $\mathrm{CuK}_{\alpha}$ radiation at a scanning rate of $0.05 \%$. The powder morphology was studied by scanning electron microscope (FEI, Quanta 200).

The estimation of oxygen and hydrogen in silver powders was carried out through inert gas fusion technique. The carbon contents of the powders were measured using a carbon analyser employing thermal conductivity detection technique.

\section{Results and discussion}

Though the solubility of silver nitrate in glycerol at room temperature is low, it increased with temperature leading to a blackish solution. Complete dissolution of the silver nitrate took place before reduction by glycerol into metallic silver. The dissolution of silver nitrate completed at a temperature of $70^{\circ} \mathrm{C}$. The blackish colour of the solution may be attributed to the nucleation of colloidal silver particles which during heating grows to form well crystalline silver powder. The precipitation of silver from the solution started at $140^{\circ} \mathrm{C}$ and went to completion at $175^{\circ} \mathrm{C}$. This was followed by vigorous boiling of the solution at the same temperature. The silver powder precipitated out in the form of soft sponge type mass that floated at the top of the solution.

The total incubation period for the precipitation of silver powder from the solution was less than an hour, which is

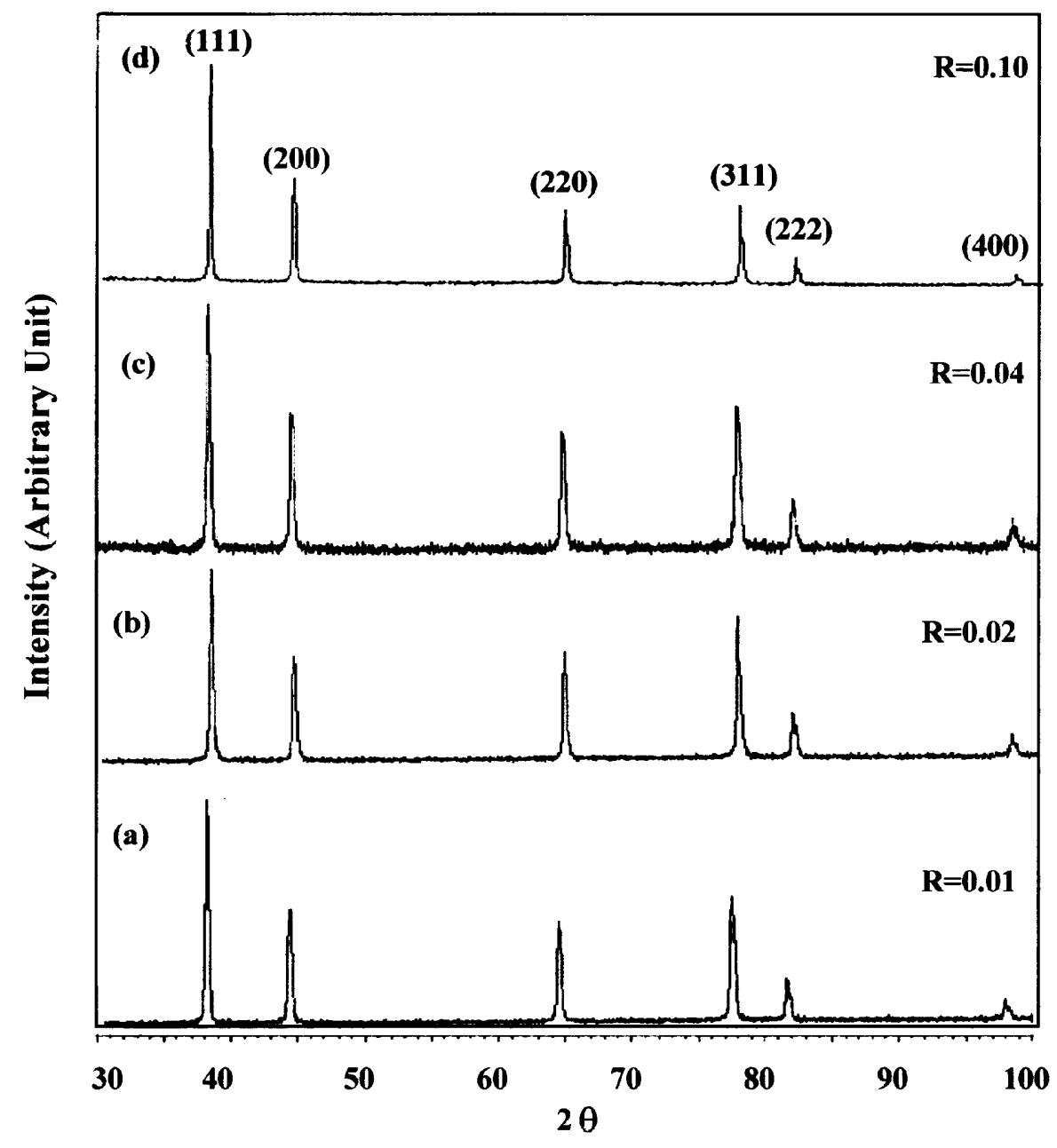

Figure 1. (a)-(d) XRD patterns of silver powders prepared through glycerol process. 
much less than other polyol processes where ethylene glycol and PVP are used as reducing agents (Silvert et al 1997). The lower value incubation period indicates the greater reducing potential of glycerol for preparation of silver powder. This is of significant importance from the commercial point of view, as a faster process would ultimately result in greater productivity.

The XRD patterns of the silver powders prepared through glycerol route are shown in figures $1 \mathrm{a}-\mathrm{d}$. The patterns reveal the diffraction peaks corresponding to $f c c$ silver phase. However, the patterns of all the powders produced through glycerol route exhibited a split in the peaks corresponding to the reflections (220), (311) and (222), respectively. Because of this splitting of XRD reflections, the estimation of crystallite size of the powders from X-ray line broadening was not attempted. To confirm the presence of split, slow scans were performed for all the samples. The typical XRD pattern taken at slow scan between $2 \theta 63.5$ and $65.5^{\circ}$ on the silver powder
$(R=0 \cdot 01)$ is shown in figure 2 . The pattern exhibits a split in the diffraction peak that may correspond to (220) reflection of cubic phase (1.444 $\AA$ ) and (110) reflection of hexagonal phase $(1.44 \AA)$. The first two strongest reflections of hexagonal allotrope of silver are $1.44 \AA$ (110) and $1.24 \AA$ (201) (ICDD No. 04-0783 and 41-1402). These reflections are very close to (220) and (311) reflections of cubic allotrope of silver having the inter planar spacing of 1.445 and $1.231 \AA$, respectively. The listing of strongest reflections of cubic and hexagonal silver is given in table 1 . The peak position and the inter planar spacing of different X-ray reflections of the silver powder produced through this process are given in table 2 . The intensities of different reflections of XRD pattern suggest that the powder is composed of majority cubic phase along with minor hexagonal phase. Sterling and Gallant (1973) first reported the presence of hexagonal allotrope in silver powder prepared by reduction of silver nitrate by starch. However, no report is available in literature on

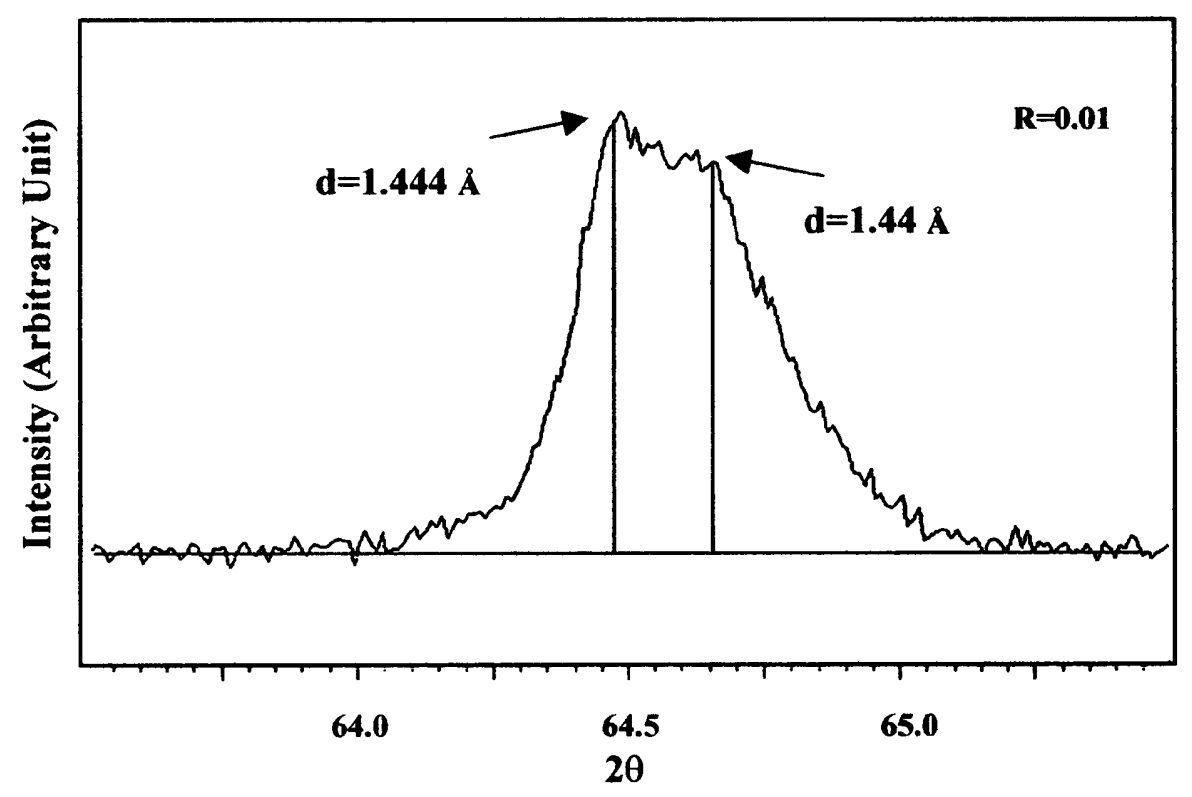

Figure 2. XRD pattern of silver powder prepared through glycerol process taken at slow scan.

Table 1. X-ray reflection of two allotropes of silver.

\begin{tabular}{lcccccc}
\hline \multicolumn{3}{c}{$\begin{array}{c}\text { Ag (cubic) } \\
\text { ICDD No. 04-0783 }\end{array}$} & & \multicolumn{3}{c}{ Ag (hexagonal) } \\
\cline { 1 - 3 } \cline { 5 - 7 }$d(\AA)$ & Intensity & $(h k l)$ & & $d(\AA)$ & Intensity & $(h k l)$ \\
\hline $2 \cdot 359$ & 100 & 111 & & $1 \cdot 44$ & 100 & 110 \\
$2 \cdot 044$ & 40 & 200 & & $1 \cdot 24$ & 100 & 201 \\
$1 \cdot 445$ & 25 & 220 & & $1 \cdot 17$ & 90 & 203 \\
$1 \cdot 231$ & 26 & 311 & & $2 \cdot 00$ & 80 & 103 \\
$1 \cdot 1796$ & 12 & 222 & & $2 \cdot 50$ & 60 & 004 \\
$1 \cdot 022$ & 4 & 400 & & $1 \cdot 00$ & 20 & 0010 \\
\hline
\end{tabular}


the presence of hexagonal allotrope in the silver powder prepared through polyol route using ethylene glycol as reducing agent.

The SEM photomicrographs of the silver powders produced through this route are shown in figures 3 and 4 .

Table 2. XRD pattern of silver powder prepared through glycerol route $(R=0 \cdot 04)$.

\begin{tabular}{lc}
\hline$d(\AA)$ & Intensity \\
\hline $2 \cdot 353$ & 100 \\
$2 \cdot 039$ & 43 \\
1.443 & 28 \\
1.439 & 16 \\
$1 \cdot 23$ & 29 \\
$1 \cdot 228$ & 16 \\
$1 \cdot 179$ & 9 \\
$1 \cdot 175$ & 4 \\
1.021 & 3 \\
\hline
\end{tabular}
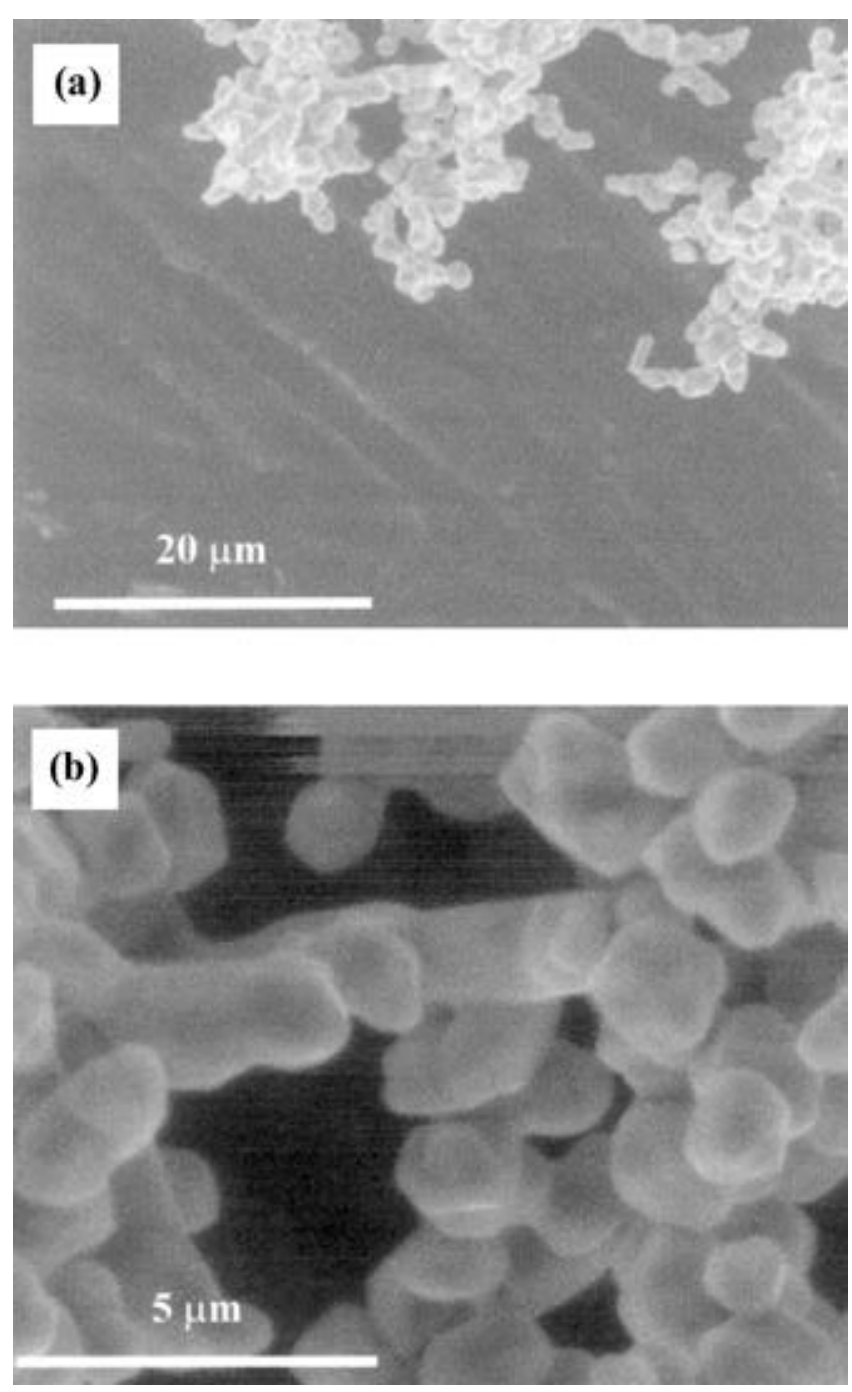

Figure 3. (a)-(b) SEM photomicrographs of silver powders produced through glycerol process using $R=0 \cdot 01$.
Figures $3 \mathrm{a}$ and $\mathrm{b}$ show photomicrographs of the powder prepared using $R=0 \cdot 01$. The micrographs reveal that the powder is made up of particles of narrow size distribution having an average particle size of $1.5 \mu \mathrm{m}$. Figures $4 \mathrm{a}$ and b show photomicrographs of the silver powder prepared through glycerol route using a molar ratio $(R)$ of 0.02 . The powder is spheroidal having narrow size distribution. The average particle size of the powder is $2 \cdot 24 \mu \mathrm{m}$.

The typical chemical analysis of silver powder produced through this route is given in table 3 . It can be seen that the total level of impurities associated with the powder is less than $0 \cdot 1 \mathrm{wt} \%$.

The above results suggest that powders produced through this route exhibit a very high purity and a good crystallinity. The particles have a regular and polyhedric shape i.e.
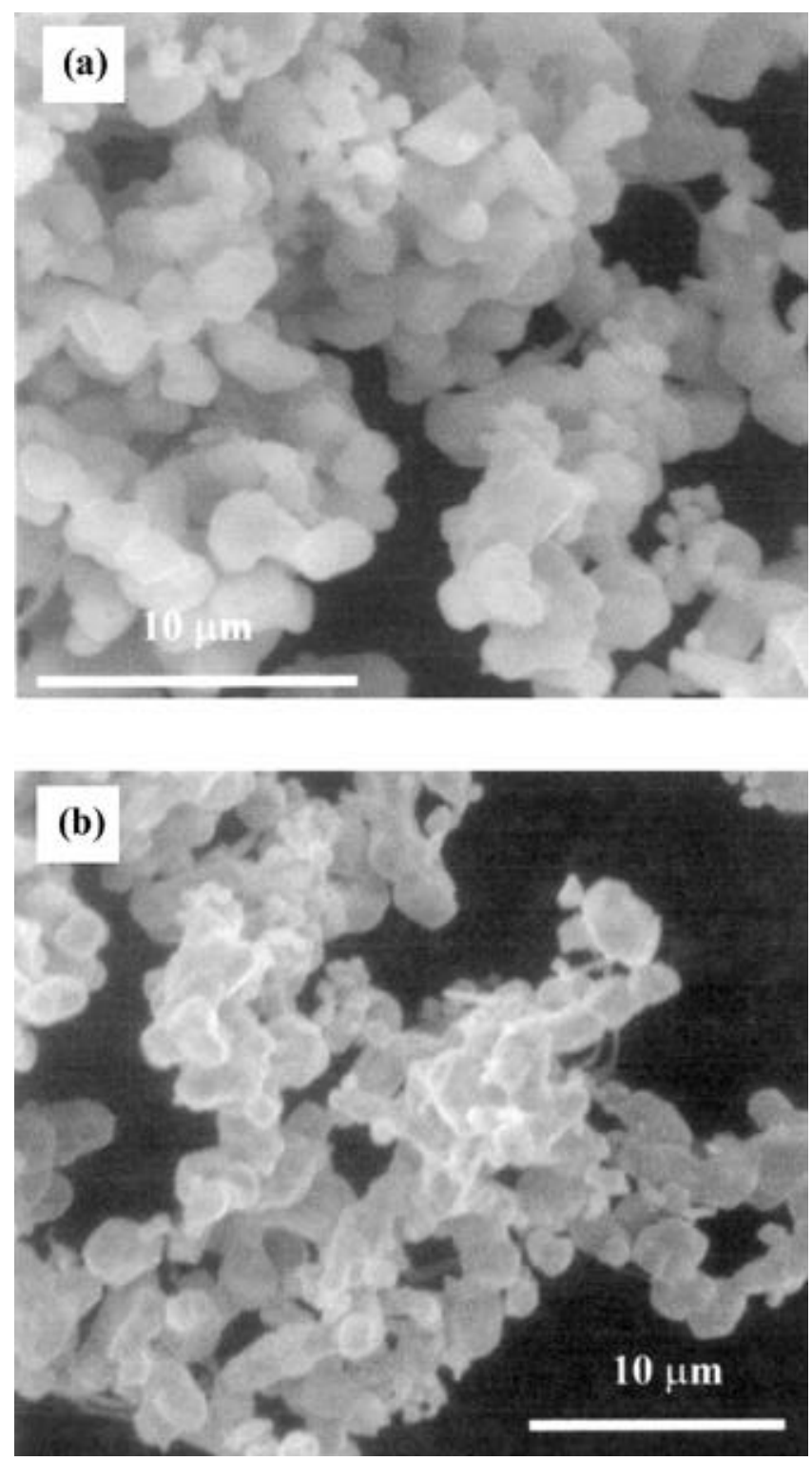

Figure 4. (a)-(b) SEM photomicrographs of silver powders produced through glycerol process using $R=0.02$. 
Table 3. Typical chemical analysis of silver powder produced through glycerol process.

\begin{tabular}{llll}
\hline Element & Carbon & Oxygen & Hydrogen \\
\hline $\mathrm{Wt} \%$ & $<0.040$ & $<0.035$ & $<90 \mathrm{ppm}$ \\
\hline
\end{tabular}

their growth is isotropic. One of the basic advantages of this process is the quantitative precipitation of silver particles from the solution leading to a process yield $>99 \%$.

The average particle size of the powder produced through this route can be controlled through the molar ratio of metal salt and glycerol $(R)$. Working under similar experimental conditions, silver powders were prepared using increasing molar ratio of $\mathrm{AgNO}_{3}$ and glycerol. Increasing the value of $R$ from $0 \cdot 01$ to $0 \cdot 1$ causes an increase in the particle size of the powder from 1.5 to $11 \mu \mathrm{m}$.

\section{Conclusions}

A simple energy efficient process has been described for preparation of silver powder starting from silver nitrate as a precursor for silver. It essentially consists of dissolving the silver precursor in glycerol followed by reduction and precipitation from the solution. The process yields well-crystallized silver powder having purity $>99.9 \%$. The particles have a regular and polyhedral shape that seems to indicate an isotropic growth of the particles. The average particle size of the powder has been found to depend on the ratio of silver nitrate and glycerol. The XRD analysis of the powder indicates the presence of hexagonal allotrope of silver as minor phase.

\section{References}

Bonet F, Tekaia-Elhsissen K and Sarathy K V 2000 Bull. Mater. Sci. 23165

Brauer G 1965 Handbook of preparative inorganic chemistry (New York: Academic Press) 2nd ed. p.1029

Brust M, Walker M, Bethell D, Schffrin D J and Whyman R 1994 J. Chem. Soc. Chem. Commun. 801

Chou K-S and Ren C-Y 2000 Mater. Chem. Phys. 64241

Duff D G, Baiker A and Edwards P P 1993 J. Chem. Soc. Chem. Commun. 96

Fievet F, Lagier J P, Blin B, Beaudoin B and Figlarz M 1989 Solid State Ionics 32/33 198

Isabel P-S and Luis L M M 1999 Langmuir 15648

Larry J R, Rosenberg R M and Uhler R G 1980 IEEE Trans. Compon. Hybrids Manuf. Technol. CHMT-3 211

Rao C N R 1994 Chemical approaches to the synthesis of inorganic materials (New York: John Wiley \& Sons)

Silvert P Y, Urbina R H and Elhsissen D T 1997 J. Mater. Chem. 7293

Sinha A and Sharma B P 2002 Mater. Res. Bull. 37407

Sterling C and Gallant D 1973 Micron 4426 